\title{
The Fermilab Main Injector Neutrino Program
}

\author{
Jorge G. Morfín \\ Fermi National Accelerator Laboratory,P.O. Box 500, Batavia, IL,60510
}

\begin{abstract}
The NuMI Facility at Fermilab provides an extremely intense beam of neutrinos making it an ideal place for the study of neutrino oscillations as well as high statistics (anti)neutrinonucleon/nucleus scattering experiments. The MINOS neutrino oscillation $v_{\mu}$ disappearance experiment is currently taking data and has published first results. The NOvA $v_{e}$ appearance experiment is planning to begin taking data at the start of the next decade.

For the study of neutrino scattering, the MINER $v$ A experiment at Fermilab is a collaboration of elementary-particle and nuclear physicists planning to use a fully active fine-grained solid scintillator detector. The overall goals of the experiment are to measure absolute exclusive crosssections, nuclear effects in $v-\mathrm{A}$ interactions, a systematic study of the resonance-DIS transition region and the high- $\mathrm{x}_{B j}$ - low $\mathrm{Q}^{2}$ DIS region.
\end{abstract}

Keywords: neutrino, scattering, oscillation PACS: $13,15,14,6,25.3,95.55$

\section{THE FERMILAB NUMI FACILITY}

The Fermilab NuMI (Neutrinos at the Main Injector) facility consists of the technical beamline components including target, two magnetic focusing horns, evacuated decay pipe, monitoring devices, shielding and the underground facilities to contain the beamline components. A large, on-site experimental detector hall $\sim 100$ meters underground currently contains the MINOS near detector. It will also house the MINERvA detector, just upstream of the MINOS near detector, and the NOVA near detector at an upstream off-axis location.

Two parabolic magnetic horns are pulsed with $200 \mathrm{kA}$ of current to focus charged hadrons $\left(\pi^{+}\right.$and $\left.\mathrm{K}^{+}\right)$into a $670 \mathrm{~m}$ long decay pipe that ends with an aluminum and steel absorber. Just downstream of the absorber, $240 \mathrm{~m}$ of Dolomite is used to range out muons before the $v$ beam enters the Near Detector Hall.

The neutrino energy distribution of the NuMI beam can be chosen by changing the distance of the target and second horn with respect to the first horn, as in a zoom lens, or, with reduced intensity but quicker tuning time, by simply varying the distance of target from the first horn and leaving the second horn in a fixed position. Depending on the chosen configuration, event rates in the near hall detectors will vary from $60 \mathrm{~K}$ in the low energy (LE) configuration to $520 \mathrm{~K}$ in the high energy (HE) configuration per ton of detector and $10^{20}$ protons on target (POT). At the far detector site in Soudan, Minnesota, the expected event rate in the $5.4 \mathrm{kT}$ MINOS far detector is (assuming no oscillations) $\sim 275$ per $10^{20}$ protons on target (POT) in the LE configuration. For the MINOS experiment and the start of the MINERvA experiment, the beamline will be operating mainly in its lowest possible neutrino energy configuration to be able to reach desired low values of $\delta m^{2}$ for MINOS. For the proposed NOvA experiment, the beam

\footnotetext{
CP917, Particles and Fields, edited by H. Castilla Valdez, J. C. D'Olivo, and M. A. Perez (C) 2007 American Institute of Physics 978-0-7354-0427-4/07/\$23.00
} 
will be operating in the full ME configuration.

The Main Injector is now delivering protons to MINOS at a rate equivalent to around $2.0-2.5 \times 10^{20}$ POT/year or $\sim 200 \mathrm{~kW}$. Upgrades to the Main Injector and other components of the Fermilab accelerator complex will increase the NuMI beam power before and during the planned operation of MINER $v$ A and NOvA. The current phase, Proton Plan I, includes upgrades to the MI RF system and other components leading to a maximum power of $430 \mathrm{~kW}$. Further upgrades proposed for after the end of TeVatron collider operations use the existing anti-proton Recycler and Accumulator rings as proton accumulators in the MI injection phase, to achieve up to $1 \mathrm{MW}$ of beam power.

\section{THE MINOS EXPERIMENT [2]}

The MINOS experiment ${ }^{1}$ tests the oscillation hypothesis by making two measurements of a beam of $v_{\mu}$ produced in the NuMI beam at Fermilab. The first measurement occurs at the Near Detector (ND) located onsite at Fermilab and the second measurement is made at the Far Detector (FD) located $735 \mathrm{~km}$ away in the Soudan Underground Mine in Soudan, Minnesota, USA. MINOS extracts the oscillation parameters by comparing the reconstructed energy spectra of the $v_{\mu}$ at the ND and FD.

\subsection{The MINOS Detectors}

The MINOS detectors are steel-scintillator tracking calorimeters with toroidal magnetic fields averaging $1.3 \mathrm{~T}$. The steel planes are $2.54 \mathrm{~cm}$ thick and the scintillator is mounted to the steel. The scintillator planes are made of $4.1 \mathrm{~cm}$ wide and $1 \mathrm{~cm}$ thick strips. The strips in each plane are rotated $45^{\circ}$ from the vertical and the strips in successive planes are rotated $90^{\circ}$ from each other. The light produced in the scintillator is collected in wavelength shifting fibers embedded in the scintillator. The fibers transport the light to multi-anode photomultiplier tubes (PMTs).

The detectors were made as similar as possible in order to cancel the majority of the uncertainties in the neutrino interaction modeling and detector response. The main design differences between the two detectors are due to the much higher rate $(\sim 100$ times higher) in the ND than in the FD. The FD is $705 \mathrm{~m}$ below the surface, has a mass of $5.40 \mathrm{kton}$. The scintillator is read out at both ends of the strips and the front end readout electronics are designed to provide high precision timing information. The ND is $103 \mathrm{~m}$ below the surface and has a mass of $0.98 \mathrm{kton}$. It uses special electronics to handle the increased rate compared to the FD. The geometry of the planes optimizes containment of hadronic showers and allows for the magnetic field to be similar to that in the FD.

\footnotetext{
${ }^{1}$ My thanks to Brian Rebel, Fermilab, for providing much of the information in this section
} 


\subsection{Event Definition and Reconstruction}

The FD data were blinded until the procedures for event selection and energy spectrum prediction were defined and understood. The blinding procedure hid a substantial and unknown fraction of the events in the FD. The data in the ND were not blinded.

The energy of each neutrino interaction is found in the same way for both detectors. Muon tracks are found and their curvature in the magnetic field is fit to determine their energy. The hadronic showers are also found and their energy is determined. The events selected in both detectors were required to have visible energy, Evis, less than $30 \mathrm{GeV}$ and the events had to have a negatively charged track, a requirement chosen to select only $v_{\mu}$ interactions. A fiducial volume was defined to contain the hadronic energy of the event and reject background cosmic ray muons. The events were also required to occur within a $50 \mu$ s window surrounding the spill time.

\subsection{The Expected Far Detector Neutrino Energy Distribution}

The measured energy spectrum in the ND is used to predict the unoscillated spectrum in the FD. The method used by MINOS to predict the FD spectrum uses the ND data to measure effects such as beam modeling, neutrino interactions and detector response that are common to both detectors. The beam simulation is used to derive a transfer matrix that relates $v_{\mu}$ in the two detectors via their parent hadrons. The matrix element Mij gives the relative probability that the distribution of secondary hadrons producing the observed $v_{\mu}$ of energy $\mathrm{E}_{i}$ in the ND will produce the observed $v_{\mu}$ of energy $\mathrm{E}_{j}$ in the FD. .

\subsection{Results}

The FD data set contains a total of 215 events with Evis $<30 \mathrm{GeV}$ compared to the unoscillated expectation of $336.0 \pm 14.4$. The uncertainty is due to systematic uncertainties associated with (a) the fiducial mass calculation and POT counting accuracy (4 $\%)$, (b) the hadronic energy scale (11\%) and (c) the NC component (50\%).

The data were fit to the hypothesis of $v_{\mu}$ to $v_{\tau}$ oscillations, and the fit has $\sin ^{2}\left(2 \theta_{23}\right) \geq 0.87$ at the $68 \%$ C.L. The best fit for the mass squared difference is $\Delta m_{23}^{2}=2.74_{-0.26}^{+0.44} \times 10^{-3} \mathrm{eV}^{2} / \mathrm{c}^{4}$ with the fit probability of $8.9 \%$.

\section{THE NOVA EXPERIMENT [1]}

The proposed NOvA (NuMI Off-axis $v_{e}$ Appearance) experiment ${ }^{2}$ will search primarily for the oscillation of muon neutrinos into electron neutrinos and the corresponding mixing angle $\theta_{13}$. NOvA will use the Fermilab NuMI beam in the ME configuration

\footnotetext{
${ }^{2}$ My thanks to Peter Shanahan, Fermilab, for providing much of the information in this section
} 
over a baseline of $810 \mathrm{~km}$, and detectors at Fermilab and the Ash River site in northern Minnesota. The detector design is optimized for the identification of electrons in the final state of $v_{e}$ charged current (CC) interactions. The Far detector will be located 12 $\mathrm{km}$ from the central axis of the beam to suppress backgrounds from intrinsic beam $v_{e}$ and high-energy neutral current interactions

\subsection{The NOvA Detectors}

The design of the NOvA detector enhances identification of $v_{e} \mathrm{CC}$ events by the separation of electromagnetic and hadronic showers. This is achieved with nearly totally active detector of relatively low $\mathrm{Z} / \mathrm{A}$ ratio, allowing a high number of samples per radiation length that is not cost-prohibitive for a detector of large mass. The detectors will be composed of liquid scintillator contained in planes of an extruded PVC cell structure, read out on one end of each cell via a wavelength-shifting fiber. The basic active cell unit will be approximately $6 \mathrm{~cm}$ deep along the beam direction, and $3.8 \mathrm{~cm}$ wide along the measurement coordinate. The wavelength shifting fibers will be read out by 32-pixel Avalanche Photo-Diodes (APDs).

The Far Detector will be situated below grade, with an overburden of between 10 and 20 radiation lengths to reduce backgrounds due to cosmic rays. A Far Detector of $\sim 20$ kT total mass will comprise roughly 1300 planes approximately $15.7 \mathrm{~m}$ on a side. The Near Detector will have the same structure, although with smaller and fewer planes, and a muon ranger to compensate.

\subsection{The NOvA Physics Goals}

NOvA will have a greatly improved sensitivity to $v_{\mu}$ to $v_{e}$ oscillations over current experiments, in part due to its low $\mathrm{Z} / \mathrm{A}$ ratio and off-axis location. NOvA will have a unique level of sensitivity to matter effects, and therefore the neutrino mass hierarchy, among present and approved experiments due to its uniquely long baseline. Depending on the size of the remaining unmeasured mixing angle, $\theta_{13}$, the detection of $\mathrm{CP}$ violation in the lepton sector could be within the reach of NOvA

\section{THE MINER $v$ A EXPERIMENT [3]}

The MINER $v A$ (Main Injector ExpeRiment: $v$ A) experiment, a collaboration of elementary-particle and nuclear physicists [4], will install a fully active fine-grained solid scintillator detector in the NuMI beam. The overall goals of the experiment are to measure absolute exclusive cross-sections, study nuclear effects in $v$ - A interactions (with A varying from $\mathrm{He}$ to $\mathrm{Pb}$ ), perform a systematic study of the resonance-DIS transition region and the lower $\mathrm{Q}^{2}$ DIS region including the extraction of high- $\mathrm{x}_{B j}$ parton distribution functions. 


\subsection{The MINER $v$ A Detector}

The MINER $v$ A detector is a hybrid of a fully-active fine-grained detector and a traditional calorimeter and is made up of a number of sub-detectors with distinct functions in reconstructing neutrino interactions. The fiducial volume for most analyses is the inner "Active Target" where all the material of the detector is the scintillator strips themselves. The scintillator detector does not fully contain events due to its low density and low $Z$, and therefore, the MINERvA design surrounds the scintillator fiducial volume with sampling detectors. To construct these sampling detectors, the scintillator strips are intermixed with absorbers. For example, the side and downstream (DS) electromagnetic calorimeters (ECALs) have lead foil absorbers. Surrounding the ECALs are the hadronic calorimeter (HCAL) where the absorbers are steel plates. On the sides of the detector the outer detector (OD) plays the role of the HCAL. In the upstream end of the detector are the nuclear targets of pure $\mathrm{C}, \mathrm{Fe}$ and $\mathrm{Pb}$ as well as a LHe target. The He target vessel is directly upstream of the main MINER $v$ A detector. Upstream of the detector and LHe target vessel is a veto of steel and scintillator strips to shield MINER $v A$ from incoming soft particles produced upstream in the hall. A complete description of MINERvA is found in the proposal [3] and TDR [5].

The core active element will be extruded triangular-shaped scintillator strips read out via wavelength-shifting fibers. Readout of the fibers will be done with multi-anode photomultiplier tubes (MAPMTs), connected to the wavelength shifting fibers via an optical cable system.

There are three distinct orientations of strips in the inner detector and veto, separated by $60^{\circ}$, and labeled X, U, V. A single module of MINER $v A$ has two X layers to seed two-dimensional track reconstruction, and one each of the $U$ and $V$ layers to reconstruct three-dimensional tracks.

Monte Carlo studies of this detector and subsequent prototype studies have confirmed that light-sharing with the triangular-shaped scintillator extrusions $(3.1 \mathrm{~cm}$ base and 1.7 $\mathrm{cm}$ height) yield reconstructed point resolution of just under $3 \mathrm{~mm}$. The electromagnetic $\left(\pi^{0}\right)$ energy resolution is $6 \% / \operatorname{sqrt}\left(E_{e m}\right)$ while the hadron energy resolution is $4 \%+$ $18 \% / \mathrm{sqrt}\left(E_{\text {had }}\right)$.

\subsection{Overview of the MINERvA Physics Program}

For a four-yeart run with 1-year of LE running parasitically with MINOS and 3-years of ME running parasitically with NOvA we expect a total of $9.0 \mathrm{M}$ in the 3-ton fiducial volume of the active scintillator target and a total of another 5.5M total events on the four nuclear targets of the MINERvA experiment.

The high-statistics studies listed below are important for both the particle and nuclear physics communities, providing information complementary to JeffersonLab charged lepton studies in the same kinematic range

- Precision measurement of the quasi-elastic neutrino-nucleus cross-section, including its $E_{v}$ and $q^{2}$ dependence, and study of the nucleon axial form factors. Over $800 \mathrm{~K}$ events are expected in the fiducial volume during the four-year MINERVA 
run.

- Determination of cross-sections in the resonance-dominated region for both neutral-current (NC) and charged-current (CC) interactions, including study of isospin amplitudes, measurement of pion angular distributions, isolation of dominant form factors, and measurement of the effective axial mass. A total of $1.7 \mathrm{M}$ one-pion events make up the low-W resonance sample.

- Clarification of the W (三 mass of the hadronic system) transition region where resonance production merges with neutrino deep-inelastic scattering, including tests of phenomenological characterizations of this transition such as quark/hadron duality. A sample of 2.1 M multi-pion events is expected with $\mathrm{W} \leq 2.0 \mathrm{GeV}$.

- Precision measurement of coherent single-pion production cross-sections, with particular attention to target $\mathrm{A}$ dependence. Coherent $\pi^{0}$ production, via the neutral current, is a significant background for next-generation neutrino oscillation experiments seeking to observe $v_{\mu} \rightarrow v_{e}$ oscillation. A sample of $89 \mathrm{~K} \mathrm{CC}$ events is expected off carbon. The expected NC sample is roughly half the CC sample.

- Examination of nuclear effects in neutrino interactions, including final-state modifications in heavy nuclei, by employing helium, carbon, iron and lead targets. These effects play a significant role in neutrino oscillation experiments measuring $v_{\mu}$ disappearance as a function of $E_{\nu}$. It has recently been suggested [?] that, for a given $\mathrm{Q}^{2}$, shadowing can occur at much lower energy transfer $(v)$ for neutrinos than for charged leptons. This effect is unaccounted for in neutrino event generators. With sufficient $\bar{v}$ running, a study of flavor-dependent nuclear effects can also be performed. Due to the different mix of quark flavors, this is another way in which neutrino and charged-lepton nuclear effects differ. MINERvA will collect over $\mathbf{0 . 6}$, 0.4, 2.0 and 2.5 $\mathrm{M}$ CC events off $\mathrm{He}, \mathrm{C}, \mathrm{Fe}$ and $\mathrm{Pb}$ targets respectively in addition to the carbon of the scintillator.

- Study of nuclear effects on $\sin ^{2} \theta_{W}$ measurements, and the NC/CC ratio for different nuclear targets.

- With a sample of over 4.3 M CC DIS events, a much-improved measurement of the parton distribution functions, particularly at large $\mathrm{x}_{B j}$, will be possible using a measurement of all three $v$ structure functions. Although we expect over $150 \mathrm{~K} \mathrm{CC}$ $\bar{v}$ events in the four year MINER $v$ A $v$ run, an additional dedicated $\bar{v}$ run would be required to measure the three $\bar{v}$ structure functions with similar precision.

- Examination of the leading exponential contributions of perturbative QCD.

- With nearly $240 \mathrm{~K}$ fully reconstructed exclusive events, precision measurement of exclusive strange-production channels near threshold. This will significantly improve our knowledge of backgrounds in nucleon-decay searches. Also, determination of $V_{u s}$, and searches for strangeness-changing neutral-currents and candidate pentaquark resonances will be undertaken Measurement of hyperonproduction cross-sections, including hyperon polarization, is feasible with exposure of MINER $v$ A to $\bar{v}$ beams

In addition to these being extremely interesting and challenging research topics, improved knowledge in most is essential to minimizing systematic uncertainties in neutrino-oscillation experiments. 


\section{CONCLUSIONS}

The Fermilab Main Injector Neutrino Program covers the entire contemporary study of neutrino physics. There is the disappearance oscillation experiment MINOS that is currently taking data and will make the most accurate measurement of $\Delta m_{23}^{2}$. The NOvA appearance oscillation experiment will atempt to measure $\sin ^{2}\left(2 \theta_{13}\right)$ and, if possible, the sign of the mass hierarchy. The MINER $v$ A experiment is a high-statistics study of the neutrino interactions that both MINOS and NOvA use to study oscillations and will help the MI oscillation experiments minimize their systematic errors.

\section{REFERENCES}

2. E. Ables et al. [MINOS Collaboration], FERMILAB-PROPOSAL-0875 and A. Marchionni [MINOS Collaboration], FERMILAB-CONF-05-429-AD-E

1. D. S. Ayres et al. [NOvA Collaboration], arXiv:hep-ex/0503053.

3. D. Drakoulakos et al. [Minerva Collaboration], arXiv:hep-ex/0405002. http://minerva.fnal.gov/

4. The MINERvA Collaboration consists of groups from the following institutions: U Athens, U California/Irvine, CBPF/Rio de Janeiro, U Dortmund, Fermilab, Hampton U, IL Inst. Tech., Inst. for Nuc. Research - Moscow, James Madison U, U Minnesota-Duluth, Jefferson Lab, U Nacional Ingeneria de Lima, N. Illinois U, Northwestern U, Pontifica U Catolica de Lima, U Pittsburgh, U Rochester, Rutgers U, U Texas-Austin, Tufts U, William and Mary U.

5. The MINERvA Technical Design Report, 1 December 2006, http://minerva-docdb.fnal.gov/cgibin/ShowDocument?docid=700 